\title{
Hypothalamic PI3K and MAPK differentially mediate regional sympathetic activation to insulin
}

\author{
Kamal Rahmouni, Donald A. Morgan, Gina M. Morgan, Xuebo Liu, Curt D. Sigmund, \\ Allyn L. Mark, and William G. Haynes \\ Hypertension Genetics Specialized Center of Research, Cardiovascular Research Center, and Department of Internal Medicine, \\ University of lowa, lowa City, lowa, USA.
}

\begin{abstract}
The action of insulin in the central nervous system produces sympathetic nervous system activation (also called sympathoactivation), although the neuronal intracellular mechanisms that mediate this are unclear. We hypothesized that PI3K and MAPK, the major pathways involved in insulin receptor signaling, mediate sympathetic nerve responses to insulin. Intracerebroventricular administration of insulin in rat increased multifiber sympathetic nerve activity to the hindlimb, brown adipose tissue (BAT), adrenal gland, and kidney. Ex vivo biochemical studies of mediobasal hypothalamic tissue revealed that insulin stimulated the association of insulin receptor substrate-1 with the $\mathrm{p} 85 \alpha$ subunit of $\mathrm{PI} 3 \mathrm{~K}$ and also tyrosine phosphorylation of $\mathrm{p} 42$ and $\mathrm{p} 44$ subunits of MAPK in the hypothalamus. In order to determine whether PI3K and/or MAPK were involved in insulinmediated sympathoactivation, we tested the effect of specific inhibitors of PI3K (LY294002 and wortmannin) and MAPK (PD98059 and U0126) on regional sympathetic responses to insulin. Interestingly, regional sympathoactivation to insulin was differentially affected by blockade of PI3K and MAPK. Inhibition of PI3K specifically blocked insulin-induced sympathoactivation to the hindlimb, while inhibition of MAPK specifically blocked insulin-induced sympathoactivation to BAT. Sympathoactivation to corticotrophin-releasing factor, however, was not affected by inhibition of PI3K and MAPK. These data demonstrate that PI3K and MAPK are specific and regionally selective mediators of the action of insulin on the sympathetic nervous system.
\end{abstract}

\section{Introduction}

There is now compelling evidence supporting a role for insulin in regulation of the sympathetic nervous system. For example, infusion of insulin during euglycemic clamping significantly increases plasma catecholamine concentrations and regional "spillover" (1-4). Hyperinsulinemia-induced sympathetic nervous system activation (also called sympathoactivation) has been confirmed using direct measurement of sympathetic nerve activity (SNA). Insulin infusion augments sympathetic outflow to skeletal muscle in humans $(5,6)$ and to the hindlimb in rats $(7)$. The rise in SNA induced by brain intracerebroventricular (ICV) administration of insulin in rats (8), as well as the prevention of the SNA responses to insulin by ablation of the anteroventral third ventricle (9), suggest that the increases in SNA induced by insulin emanate from the hypothalamus. Insulin receptors have been located in several brain regions, including those area involved in sympathetic regulation such as the hypothalamus (10-12). However, the intracellular signaling pathways implicated in insulin-induced sympathoactivation remain unknown. Moreover, it is unclear whether the same mechanisms are involved in differential regional sympathetic responses to insulin.

Insulin receptor signaling depends on the activation of diverse mechanisms $(13,14)$. Activation of the insulin receptor results in

Nonstandard abbreviations used: BAT, brown adipose tissue; CRF, corticotrophinreleasing factor; CSF, cerebrospinal fluid; $\mathrm{HR}$, heart rate; ICV, intracerebroventricular; IRS, insulin receptor substrate; SHC, Src homology collagen; SNA, sympathetic nerve activity.

Conflict of interest: The authors have declared that no conflict of interest exists.

Citation for this article: J. Clin. Invest. 114:652-658 (2004)

doi:10.1172/JCI200421737. tyrosine phosphorylation of insulin receptor substrate-1 (IRS-1) through IRS-4. This allows association of IRSs to various effector molecules such as the regulatory subunit of PI3K through its Src homology 2 domain (15). Once activated, PI3K activates protein kinase $\mathrm{C}$ and a serine/threonine protein kinase, Akt/protein kinase B. Another major signaling pathway of the insulin receptor involves the cytoplasmic intermediate protein called the Src homology collagen (SHC) protein $(13,14)$. When tyrosine phosphorylated, SHC associates with the growth factor receptor-binding protein $2 /$ sonof-sevenless complex, leading to activation of the Ras/Raf pathway, which in turn triggers activation of MAPK.

The aim of the present study was to examine the molecular mechanisms involved in the effects of insulin on regional SNA. We hypothesized that PI3K and MAPK mediate sympathetic nerve responses to insulin. First, we established the effect of ICV insulin on SNA to different tissues including hindlimb, brown adipose tissue (BAT), kidney, and adrenal glands. Next, we examined biochemically whether insulin activates PI3K and MAPK in the hypothalamus. Finally, in order to determine whether PI3K and/ or MAPK were involved in insulin-mediated sympathoactivation, we tested the effect of specific inhibitors of PI3K (LY294002 and wortmannin) and MAPK (PD98059 and U0126) on regional sympathetic responses to insulin.

\section{Results}

Sympathetic nerve effects of ICV insulin. Insulin concentrations in the cerebrospinal fluid (CSF) were significantly higher in rats treated with ICV insulin than in vehicle-treated animals (Table 1). Plasma insulin, however, did not differ between the control group and ICV insulin-treated animals (Table 1). 

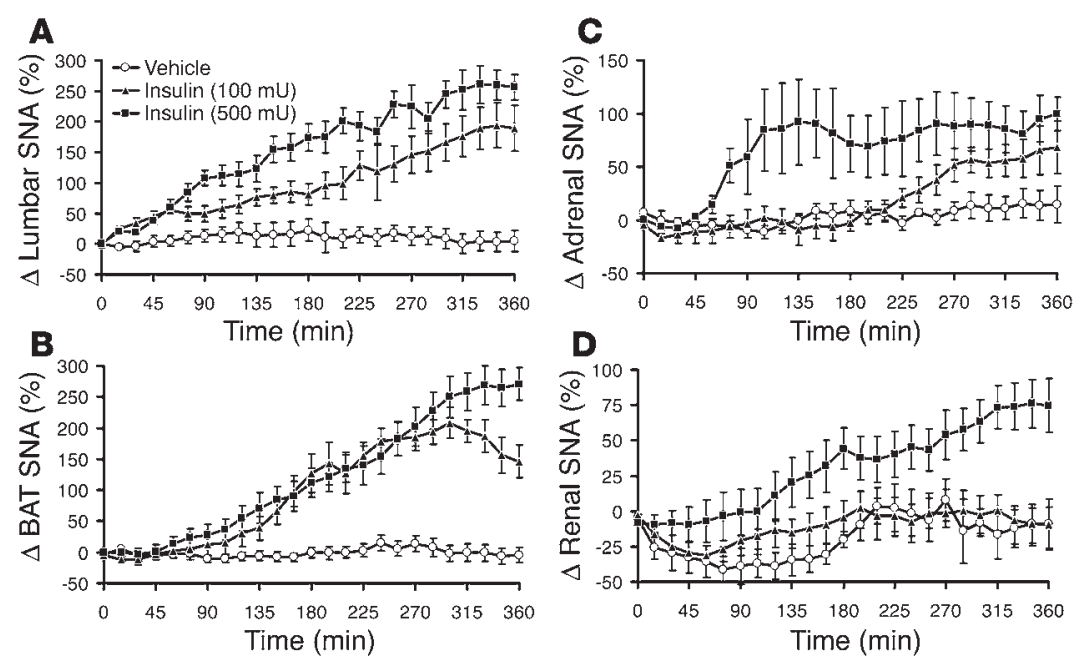

\section{Figure 1}

Effects of insulin on regional SNA. (A-D) Effects of ICV administration of insulin on multifiber SNA to hindlimb (A), BAT (B), adrenal gland (C), and kidney (D) in anesthetized Sprague-Dawley rats. $\triangle$ SNA represents the percent change from baseline. Data represent means \pm SEM of 8-18 rats per group.

$(103 \pm 47 \mathrm{mg} / \mathrm{dl}$ and $107 \pm 21 \mathrm{mg} / \mathrm{dl})$ after ICV injection. Plasma glucose was also not affected by ICV administration of insulin or vehicle (Table 1). In the group of rats treated with $500 \mathrm{mU}$ of insulin, blood glucose was $49 \pm 3$ at baseline, $42 \pm 3 \mathrm{mg} /$ $\mathrm{dl}$ at 2 hours, $47 \pm 7 \mathrm{mg} / \mathrm{dl}$ at 4 hours, and $51 \pm 6$ $\mathrm{mg} / \mathrm{dl}$ at 6 hours. Finally, to exclude the possibility of any effect of low CNS glucose levels on regional sympathoactivation to insulin, we assessed the added effect of ICV glucose administration (100

ICV administration of insulin caused a significant $(P<0.0001)$ and dose-dependent increase in lumbar SNA (Figure 1A), with $187 \% \pm$ $36 \%(P<0.01$ vs. diluent) and $257 \% \pm 21 \%(P<0.01)$ increases in the 6 th hour at doses of 100 and $500 \mathrm{mU}$, respectively. ICV insulin produced a slow increase in BAT SNA $(P<0.0001$; Figure 1B), with $171 \% \pm 24 \%(P<0.001)$ and $280 \% \pm 30 \%(P<0.001)$ increases in the 6 th hour at the lowest and highest doses, respectively. Adrenal sympathoactivation to ICV insulin was modest, with a mean increase of $90 \% \pm 18 \%$ in the 6 th hour at the highest dose $(P<0.05$; Figure $1 C)$. Renal SNA increased slowly only with the higher dose of insulin (Figure 1D). The rise in renal SNA induced by $500 \mathrm{mU}$ of insulin was $76 \% \pm 16 \%$ at the 6 th hour after the ICV injection $(P<0.01)$. While mean arterial pressure tended to increase only with the highest dose of insulin, heart rate (HR) was significantly $(P=0.0038)$ increased in insulin-treated animals (Table 1).

To exclude the possibility that the regional sympathoactivation to insulin was caused by hypoglycemia, we assessed CSF and plasma glucose after ICV administration of insulin and vehicle. CSF glucose was not significantly affected by ICV administration of insulin, as CSF glucose was comparable in rats treated with vehicle or different doses of insulin at 6 hours (Table 1). Insulin (500 mU) also failed to affect CSF glucose at 15 minutes $(127 \pm 7 \mathrm{mg} / \mathrm{dl}$ and $134 \pm 1 \mathrm{mg} / \mathrm{dl}$ in rats treated with vehicle and insulin, respectively), 30 minutes $(104 \pm 29 \mathrm{mg} / \mathrm{dl}$ and $127 \pm 14 \mathrm{mg} / \mathrm{dl})$, and 60 minutes $\mu \mathrm{g})$ to the SNA response to $500 \mathrm{mU}$ of insulin. The lumbar SNA response to insulin was preserved $(254 \% \pm 47 \% ; n=6$; $P=0.94$ vs. insulin alone) when glucose was administered with insulin.

Effects of insulin on bypothalamic PI3K and MAPK. To investigate whether insulin activates PI3K and MAPK in the hypothalamus, we tested the effects of ICV administration of $500 \mathrm{mU}$ of insulin on these enzymes in mediobasal hypothalamic tissue. We found that in vivo ICV administration of insulin caused a significant $(P<0.001)$ increase in IRS-1-associated PI3K, with a peak at $15 \mathrm{~min}$ utes (Figure 2A). ICV insulin also caused a significant $(P<0.001)$ increase in the tyrosine phosphorylation of p42 and p44 MAPK, peaking at 30 minutes, but insulin treatment did not affect total MAPK (Figure 2B). Thus, insulin activates both PI3K and MAPK pathways in the hypothalamus.

Role of PI3K in SNA responses to insulin. As shown in Figure 3, sympathoactivation to insulin was significantly attenuated by ICV administration of $5 \mu \mathrm{g}$ of the PI3K inhibitor LY294002 and was completely abolished in the presence of $0.5 \mu \mathrm{g}$ of wortmannin. The increase in lumbar SNA induced by ICV administration of $500 \mathrm{mU}$ of insulin was $248 \% \pm 21 \%$ in presence of vehicle, $116 \% \pm 21 \%$ in presence of LY294002 $(P<0.01)$, and $23 \% \pm 19 \%$ $(P<0.001)$ in the presence of wortmannin. In presence of $1 \mu \mathrm{g}$ of LY294002, the attenuation of lumbar sympathoactivation to insulin was less pronounced $(194 \% \pm 22 \% ; n=6$; $P<0.05$ vs.

\section{Table 1}

Endocrine and hemodynamic parameters obtained from rats 6 hours after ICV administration of insulin or vehicle

\begin{tabular}{|c|c|c|c|c|c|c|}
\hline Treatment & $\begin{array}{l}\text { CSF insulin } \\
(\mathrm{ng} / \mathrm{ml})\end{array}$ & $\begin{array}{l}\text { CSF glucose } \\
(\mathrm{mg} / \mathrm{dl})\end{array}$ & $\begin{array}{l}\text { Plasma insulin } \\
\quad(\mathrm{ng} / \mathrm{ml})\end{array}$ & $\begin{array}{l}\text { Plasma glucose } \\
(\mathrm{mg} / \mathrm{dl})\end{array}$ & $\begin{array}{c}\text { Mean arterial } \\
\text { pressure }(\mathrm{mmHg})\end{array}$ & $\begin{array}{c}\text { HR } \\
(\mathrm{bpm})\end{array}$ \\
\hline \multicolumn{7}{|l|}{ ICV vehicle } \\
\hline Basal & ND & ND & ND & $55 \pm 3$ & $134 \pm 6$ & $355 \pm 9$ \\
\hline 6 hours & $0.12 \pm 0.05$ & $54 \pm 4$ & $2.3 \pm 0.6$ & $48 \pm 5$ & $128 \pm 6$ & $378 \pm 12$ \\
\hline \multicolumn{7}{|c|}{ ICV insulin (100 mU) } \\
\hline Basal & ND & ND & ND & $64 \pm 5$ & $131 \pm 4$ & $345 \pm 9$ \\
\hline 6 hours & $114.9 \pm 30.8^{A}$ & $53 \pm 4$ & $1.7 \pm 0.3$ & $62 \pm 5$ & $130 \pm 4$ & $373 \pm 11^{B}$ \\
\hline \multicolumn{7}{|c|}{ ICV insulin (500 mU) } \\
\hline Basal & ND & ND & ND & $49 \pm 3$ & $127 \pm 6$ & $355 \pm 10$ \\
\hline 6 hours & $195.5 \pm 31.2^{A}$ & $58 \pm 8$ & $1.9 \pm 0.3$ & $51 \pm 6$ & $132 \pm 5$ & $401 \pm 11^{B}$ \\
\hline
\end{tabular}

Data are means \pm SEM of $6-30$ rats per group. bpm, beats per minute; ND, no data. ${ }^{A} P<0.01$ versus vehicle; ${ }^{B} P<0.05$ versus basal. 
A

IRS-1

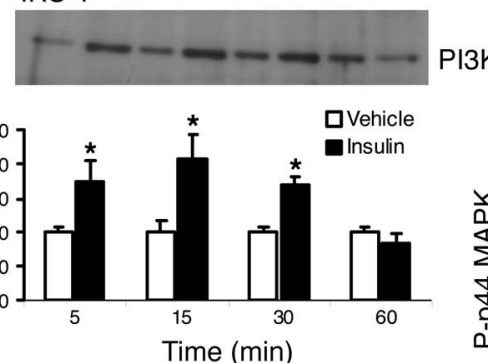

B
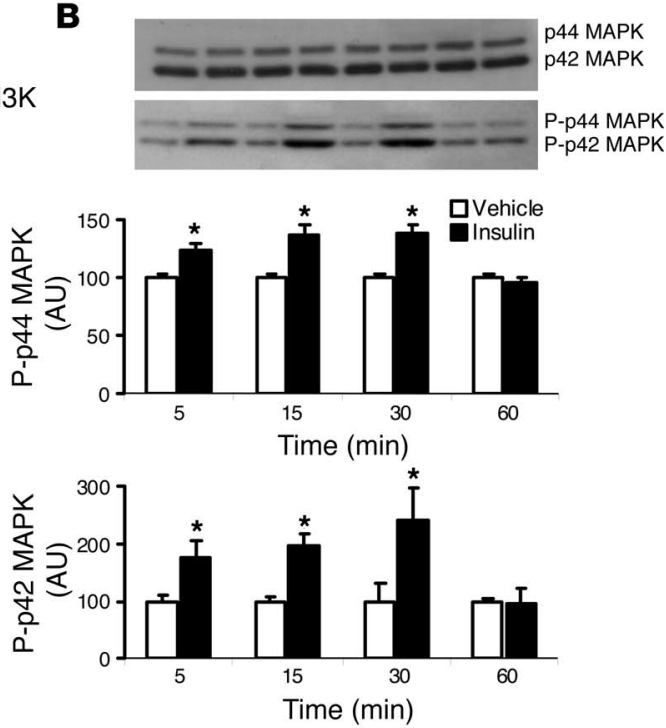

Figure 2

Insulin activates PI3K and MAPK in the hypothalamus. Rats were killed at the indicated time points after ICV administration of $500 \mathrm{mU}$ of insulin or vehicle, and total proteins were extracted from the mediobasal hypothalamus and assayed. (A) Effect of ICV administration of insulin on the interaction of IRS-1 and the p85 subunit of PI3K in the mediobasal hypothalamus. Top, Western blot of p85 PI3K immunoprecipitated with IRS-1. Bottom, densitometric analysis of the immunoreactive bands for p85 PI3K immunoprecipitated with IRS-1, expressed as change relative to that of vehicle-treated group. (B) Effect of ICV administration of insulin on the total MAPK (p42 and p44 MAPK) and phosphorylated forms of MAPK (P-p42 and P-p44 MAPK) in the mediobasal hypothalamus. Top, Western blot of the total and phosphorylated forms of MAPK. Bottom, densitometric analysis of the immunoreactive bands for phosphorylated form of MAPK, expressed as change relative to that of vehicle-treated group. Data represent means \pm SEM of 4 rats per group. ${ }^{*} P<0.001$ versus the vehicle-treated group. $\mathrm{AU}$, arbitrary units.

insulin), indicating that the effect of PI3K inhibitors on the lumbar SNA response to insulin is dose dependent. In contrast, the increase in BAT, adrenal, and renal SNA induced by insulin was not significantly altered by pretreatment with LY294002 or wortmannin (Figure 3). PI3K inhibitors had no effect on baseline SNA when given alone.

Administration of LY294002 or wortmannin with insulin or vehicle did not significantly affect mean arterial pressure, HR, or plasma insulin (data not shown). The CSF insulin of rats receiving $5 \mu \mathrm{g}$ of LY294002 and $500 \mathrm{mU}$ of insulin was elevated $(213 \pm 33 \mathrm{ng} / \mathrm{ml})$ compared with that of rats receiving LY294002 alone $(0.10 \pm 0.04 \mathrm{ng} / \mathrm{ml})$.

Role of MAPK in SNA responses to insulin. Pretreatment with $5 \mu \mathrm{g}$ of PD98059 or $7 \mu \mathrm{g}$ of U0126, which had no effect alone, abolished the increase in SNA to BAT (Figure 4). Insulin increased BAT SNA by $280 \% \pm 26 \%$ in presence of vehicle, but failed to affect BAT SNA in presence of PD98059 $(47 \% \pm 13 \% ; P<0.001)$ or U0126 $(37 \% \pm 8 \%$; $P<0.001)$. The effect of MAPK inhibitors on BAT sympathoactivation to insulin appears to be dose dependent, as the inhibition of BAT SNA in response to insulin was less pronounced in presence of $1 \mu \mathrm{g}$ of PD98059 (198\% $\pm 26 \% ; n=6 ; P<0.001$ vs. insulin). In contrast, lumbar, adrenal, and renal sympathoactivation to insulin were not significantly affected by PD98059 or U0126 (Figure 4B).

Administration of the MAPK inhibitor PD98059 with insulin or vehicle did not alter mean arterial pressure, HR, or plasma insulin (data not shown). CSF insulin was increased in the rats treated with a combination of $5 \mu \mathrm{g}$ of PD98059 and insulin
$(230 \pm 98 \mathrm{ng} / \mathrm{ml})$ compared with that of rats treated with PD98059 alone $(0.15 \pm 0.06 \mathrm{ng} / \mathrm{ml})$.

Effects of PI3K and MAPK blockade on SNA response to corticotrophin-releasing factor. In line with a previous report (16), ICV administration of $5 \mu \mathrm{g}$ of corticotrophin-releasing factor (CRF) caused a substantial and significant $(P<0.01)$ increase in SNA to hindlimb and BAT (Figure 5). Pretreatment with the PI3K inhibitors LY294002 $(5 \mu \mathrm{g})$ and wortmannin $(0.5 \mu \mathrm{g})$ did not affect the increase in lumbar SNA induced by CRF (Figure 5A). Similarly, pretreatment with the MAPK inhibitors PD98059 $(5 \mu \mathrm{g})$ and U0126 $(7 \mu \mathrm{g})$ did not alter the BAT SNA response to CRF (Figure 5B).

\section{Discussion}

Our data demonstrate that CNS administration of insulin in the rat increases sympathetic nerve outflow to the hindlimb, BAT, kidney, and adrenal gland. More surprisingly, our data show that contrasting molecular mechanisms are involved in regional sympathoactivation induced by insulin. The increase in sympathetic outflow to thermogenic BAT is mediated by MAPK, while the sympathoexcitatory effect of insulin to the hindlimb is mediated by PI3K. However, neither of these signaling pathways appears to be involved in insulin-mediated sympathoexcitation to the kidney and adrenal gland. These data confirm the hypothesis that PI3K and MAPK are major intracellular mediators in the regulation of sympathetic outflow by insulin.

Studies from several laboratories, including our own, have shown that insulin produces nonuniform regional sympathetic nerve responses $(7,8,17,18)$. In rats, hyperinsulinemia was found to increase sympathetic activity to the hindlimb but not to other tissues $(7,8)$. In contrast, our present data demonstrate that insulin increased hindlimb SNA, but also increased SNA to BAT, adrenal gland, and kidney. While the increase in hindlimb SNA was fast, beginning minutes after ICV administration of insulin, the SNA responses of other nerves were slower and required 1-2 hours to start. This slow increase in SNA to BAT, kidney, and adrenal gland could explain the contrasting findings between our study and previous reports in which the effect of insulin on SNA was assessed only during the first 2 hours.

Insulin-induced sympathoactivation to thermogenic BAT is consistent with the role of insulin in the regulation of body weight and energy homeostasis. Indeed, ICV administration of insulin has been shown to decrease body weight in several species (19). In contrast, downregulation of insulin receptors in the hypothalamus using insulin receptor antisense oligodeoxynucleotide (20) as well as neuron-specific deletion of this receptor throughout the central nervous system (21) increases fat mass. Furthermore, the action of insulin in the central nervous system increases 

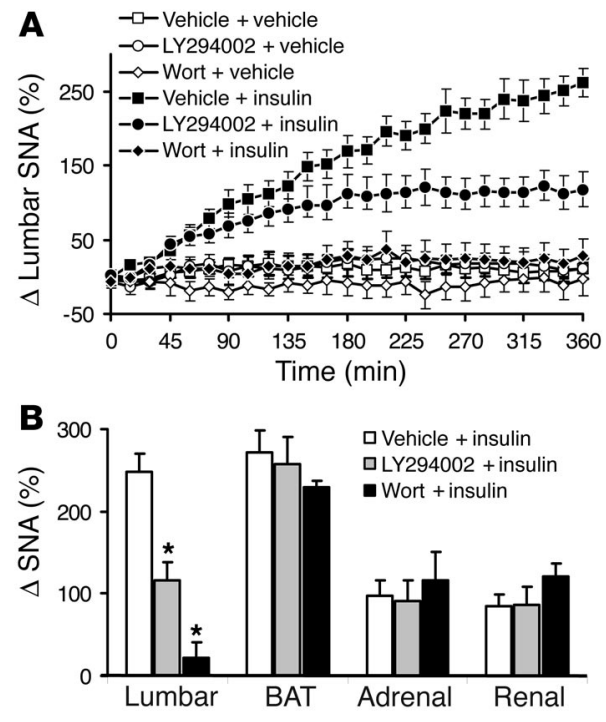

Figure 3

Effects of PI3K blockade on regional SNA responses to $500 \mathrm{mU}$ of ICV insulin. (A) Attenuated lumbar SNA response to insulin in the presence of the PI3K inhibitors LY294002 (5 $\mu$ g; ICV) and wortmannin (Wort; 0.5 $\mu \mathrm{g}$; ICV). (B) Blockade of SNA response to insulin with PI3K inhibitors is selective for the hindlimb, as PI3K inhibitors have no effect on BAT, adrenal, or renal SNA responses to insulin. Data represent means \pm SEM of 8-18 animals per group. ${ }^{*} P<0.001$ versus the vehicle + insulin group.

thermogenesis (22) and stimulates the level of uncoupling protein in BAT (23). The increase in SNA observed after CNS administration of insulin is not secondary to an effect of insulin on either CSF or blood glucose concentrations. Indeed, CSF and plasma glucose levels were similar in insulin- and vehicle-treated animals, while the rise in SNA after administration of ICV insulin was dose related. Additionally, lumbar sympathoactivation to insulin was not altered by central administration of glucose. However, the absence of effect of ICV insulin on plasma glucose in our study here is in contrast with the previously reported role of CNS insulin in the regulation of plasma glucose. Indeed, the action of insulin in the hypothalamus has been reported to decrease glucose production (24) and plasma glucose concentrations (25). Although the reasons for the discrepancy between our study and those previous reports remain unknown, the presence of prolonged anesthesia has been shown to inhibit the fall in plasma glucose induced by the CNS action of insulin (26).

The sympathetic effects of insulin on organs involved in the cardiovascular regulation, such as the kidney, are also consistent with the "pro-hypertensive" action of insulin $(27,28)$. This hormone is also known to stimulate sodium retention, presumably via stimulation of renal nerve outflow $(18,29)$. In contrast, in our studies ICV insulin caused only a slight increase in arterial pressure despite the robust increase in SNA, perhaps because of the presence of anesthesia (30). The absence of a significant pressor effect of insulin in our study here may also relate to the relatively short study duration, because chronic infusion of insulin has been shown to increase blood pressure in the rat (28). In the so-called "insulin hypothesis of hypertension" $(18,31)$, the sympathetic effects of chronic hyperinsulinemia have been suggested to link insulin resistance, cardiovascular mortality, and high blood pressure.
Exploration of the mechanisms by which insulin controls the sympathetic nervous system may offer insights into CNS mechanisms of obesity, insulin resistance, and cardiovascular diseases, including hypertension. Here we have demonstrated the involvement of nonuniform intracellular mechanisms in the control of regional SNA by insulin. This is consistent with the divergent signaling capacities of the insulin receptor that affect several physiological processes through different pathways $(13,14)$. In the central nervous system, Spanswick et al. (32) have shown that blockade of PI3K, but not MAPK, abolishes the neuronal hyperpolarization induced by insulin. Hypothalamic PI3K also appears to be a key component in the effects of insulin on food intake and body weight, as blockade of this enzyme prevents the anorexic and weight-reducing effects of insulin $(33,34)$. Abolition of the weight-reducing effect of insulin with PI3K inhibitors would suggest that this enzyme is central to insulin-induced thermogenesis. In contrast, our data have shown that insulin-induced sympathoactivation to thermogenic BAT is MAPK mediated. It could be that insulin-induced sympathoactivation, which was prevented with PI3K inhibitors, contributes to the thermogenic action of insulin. This would indicate that lumbar sympathoactivation represents an increase mainly in nerve fibers that subserve metabolic function. The absence of an effect of PI3K and MAPK blockade on the renal and adrenal SNA responses to insulin indicates that other intracellular signaling mechanisms mediate insulin-induced sympathoexcitation to these tissues. In support of this hypothesis is the difference in the magnitude as well as the time course of the SNA responses to insulin of the nerves serving these two tissues compared with BAT and lumbar SNA responses. The difference in the magnitude and time course of regional SNA response to insulin may also suggest that different neuronal pathways are involved in the control by insulin of regional sympathetic outflow. Central insulin modulates distinct neuropeptide systems, including proopiomelanocortin peptide and neuropeptide $\mathrm{Y}(19,35,36)$.
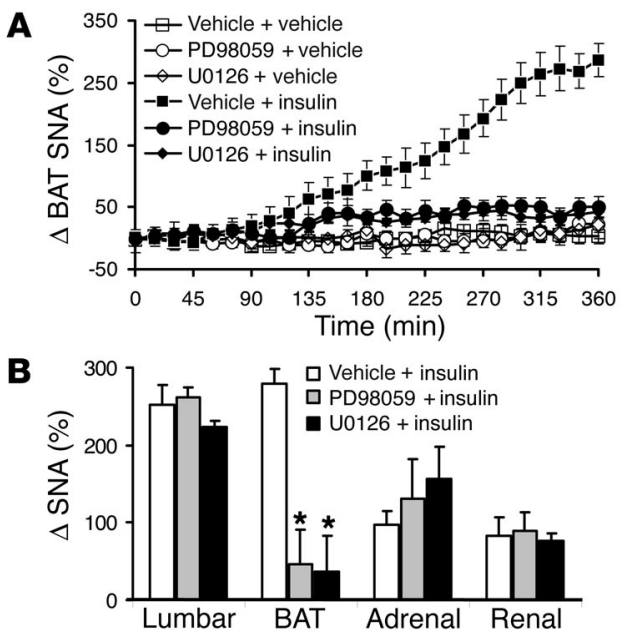

\section{Figure 4}

Effects of MAPK blockade on regional SNA responses to $500 \mathrm{mU}$ of ICV insulin. (A) Attenuated BAT SNA response to insulin in the presence of the MAPK inhibitors PD98059 (5 $\mu \mathrm{g}$; ICV) and U0126 (7 $\mu \mathrm{g}$; ICV). (B) Blockade of SNA response to insulin with MAPK inhibitors is selective for BAT, as MAPK inhibitors have no effect on lumbar, adrenal, or renal SNA responses to insulin. Data represent means \pm SEM of 8-18 animals per group. ${ }^{*} P<0.001$ versus the vehicle + insulin group. 

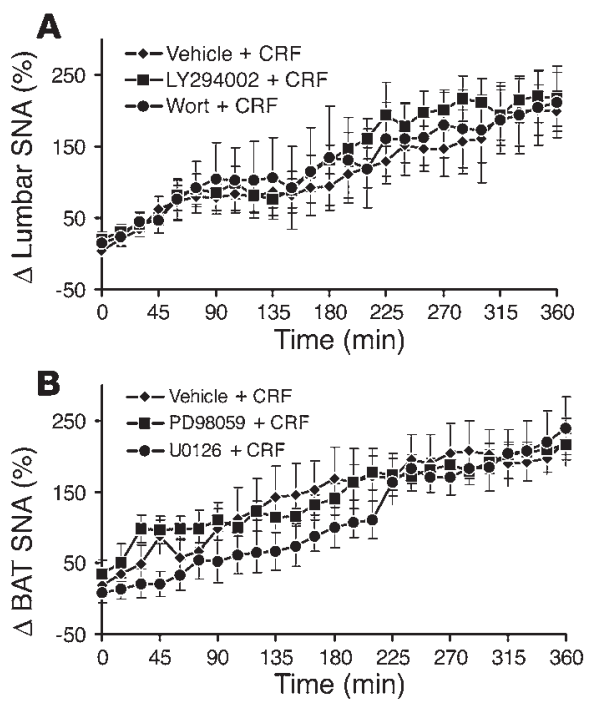

\section{Figure 5}

Effects of PI3K and MAPK blockade on lumbar and BAT SNA responses to another stimulus, CRF. (A) The PI3K inhibitors LY294002 (5 $\mu \mathrm{g}$; ICV) and wortmannin ( $0.5 \mu \mathrm{g}$; ICV) have no effect on the lumbar sympathoactivation to CRF ( $5 \mu \mathrm{g}$; ICV). (B) The MAPK inhibitors PD98059 (5 $\mu \mathrm{g}$; ICV) and U0126 (7 $\mu \mathrm{g}$; ICV) have no effect on the BAT sympathoactivation to CRF (5 $\mu \mathrm{g} ; \mathrm{ICV})$. Data represent means \pm SEM of $8-10$ rats per group.

Interestingly, the effect of insulin on the renal SNA appears to be mediated by the melanocortin system, as renal sympathoactivation to insulin is abolished in homozygous melanocortin 4 receptor-knockout mice, while heterozygous mice have an attenuated response to insulin (37). The role of the melanocortin system in the SNA response of other nerves to insulin remains unknown, however. The involvement of other neuropeptides in the control of sympathetic nervous system by insulin is also unknown.

Because PI3K and MAPK are central molecules for many cellular effects, we questioned the specificity of blockade of insulininduced sympathoactivation by inhibition of these enzymes. However, CRF-induced lumbar sympathoactivation was not affected by the presence of PI3K inhibitors. Similarly, inhibition of MAPK did not affect the insulin-induced rise in BAT SNA. Thus, the involvement of PI3K and MAPK in the control of lumbar and BAT SNA seems to be specific for insulin. Additionally, the relatively high degree of specificity of the inhibitors used in our study excludes the possibility that blockade of SNA responses to insulin may be due to a nonspecific effect of these compounds. Indeed, cell-based assays and in vitro studies have shown that both LY294002 and wortmannin inhibit PI3K far more potently than they inhibit any other related enzyme $(15,38)$. Similarly, PD98059 and U0126 appear to specifically suppress MAPK signaling, rather than suppressing any other protein kinase (38-40). These inhibitors have been widely used to suppress activation of PI3K and MAPK and to examine the physiological roles of these enzymes.

In conclusion, we have demonstrated that ICV administration of insulin substantially increases SNA to the hindlimb, BAT, kidney, and adrenal gland. The finding that ICV insulin does not alter plasma insulin levels indicates that these effects are due solely to central neural action of insulin. Our data also reveal a key role for MAPK and PI3K in the control of regional sympathetic outflow by insulin. Indeed, the increase in SNA to thermogenic BAT is mediated by MAPK, while the sympathoexcitatory effect of insulin to the hindlimb is mediated by PI3K. In contrast, none of these signaling pathways seem to mediate the effect of insulin on SNA to the kidney and adrenal gland. Our data offer the tantalizing possibility that it may be possible to selectively interfere with potentially deleterious cardiovascular sympathetic activation, while not altering thermogenic, weight-reducing sympathoexcitation. Our data also suggest that it may be possible to therapeutically modulate thermogenic SNA without altering nonthermogenic SNA.

\section{Methods}

Animals. Male Sprague-Dawley rats (Harlan Sprague-Dawley) weighing between 300 and $400 \mathrm{~g}$ were used. All rats were housed in a room at constant temperature $\left(23^{\circ} \mathrm{C}\right)$ with a 12 -hour light/dark cycle (light switched off at 18:00). Rats were fed standard rat chow (containing $18.62 \%$ protein, $6.25 \%$ fat, $4.53 \%$ fiber, and $53 \%$ nitrogen-free extracts; Harlan Teklad) and were given water ad libitum. The University of Iowa Animal Research Committee (Iowa City, Iowa, USA) approved all procedures.

Cannulae implantation. Rats were anesthetized by intraperitoneal injection of pentobarbital (Nembutal, $50 \mathrm{mg} / \mathrm{kg}$ ) and were placed in a stereotactic device (Kopf Instruments). A 23-gauge sterile guide cannula $16 \mathrm{~mm}$ in length was implanted $10^{\circ}$ from vertical into the third brain ventricle with the following coordinates relative to bregma: $-1 \mathrm{~mm}$ anteroposterior, +1.5 $\mathrm{mm}$ lateral from the midline, and $-9 \mathrm{~mm}$ dorsoventral. The ICV cannula was then fixed in place with dental cement and rats were kept in individual cages postoperatively. Cannulae were considered patent in rats that drink more than $4 \mathrm{ml}$ of water in response to carbachol injection $(50 \mathrm{ng}$; ICV). The position of the cannula was verified at the end of each experiment by histological analysis.

Surgical procedure for hemodynamic and nerve recordings. One week after ICV cannulation, rats were anesthetized with pentobarbital and a catheter was inserted in the jugular vein for maintenance of anesthesia with $\alpha$-chloralose $(50 \mathrm{mg} / \mathrm{kg} / \mathrm{h})$. We have previously shown that this anesthesia regimen does not alter the sympathetic response to different stimuli, including baroreflex stimulation and hemorrhage (41). The trachea was cannulated, and each rat was allowed to spontaneously breathe oxygenenriched air. Rectal temperature was maintained at $37.5^{\circ} \mathrm{C}$ with a temperature-controlled surgical table and a lamp. Hemodynamic parameters (mean arterial pressure and HR) were monitored through a pressure transducer connected to the catheter inserted in the femoral artery with a pressure transducer connected to MaLab data acquisition system and a Macintosh computer.

SNA to BAT, kidney, hindlimb, and adrenal gland was measured by multifiber recording. The left kidney and adrenal gland were exposed retroperitoneally through a flank incision. Under a dissecting microscope, a renal or adrenal nerve was carefully dissected free and was placed on a bipolar 36-gauge platinum-iridium electrode (Cooner Wire Co.). When optimum recording of SNA was obtained, the electrode was covered with silicone gel (World Precision Instruments Inc.). For recording BAT SNA, the BAT was exposed through a incision in the nape of the neck. A sympathetic nerve fiber innervating BAT was identified, placed on the bipolar platinum-iridium electrode, and fixed with silicone gel. Separate groups of rats were prepared for measurement of lumbar SNA. A middle abdominal incision was made and a lumbar sympathetic nerve was isolated, placed on the bipolar platinum-iridium electrode, and covered with silicone gel. In some rats, we performed simultaneous recording of SNA from two nerves serving different tissues (BAT with adrenal or renal).

Nerve electrodes were attached to a high-impedance probe (HIP-511; Grass Instruments Co.). The nerve signal was amplified 10 times with a 
Grass P5 AC preamplifier, filtered at a 100- and 1,000-Hz cutoff with a nerve traffic analysis system (model 706C; University of Iowa Bioengineering, Iowa City, Iowa, USA), and led to an oscilloscope (model 54501A; Hewlett-Packard Co.) whose cursor was positioned precisely above the background noise. The nerve traffic analyzer counted the action potentials that exceeded this threshold voltage (spikes/second). Both the counted action potentials and the renal neurogram were routed to a MacLab analog-digital converter (model 8S; AD Instruments Castle Hill) for permanent recording and data analysis on a Macintosh computer. To ensure that electrical noise was excluded in the assessment of sympathetic outflow, SNA was corrected for post-mortem background activity.

SNA studies. After surgery to measure SNA and hemodynamics was completed, animals were allowed to stabilize for 15-20 minutes. Baseline SNA for each "bed," mean arterial pressure, and HR were then measured on two occasions throughout a 10 -minute control period and were averaged to obtain a single value for this control period. In the dose-response study, arterial blood samples were also obtained at baseline for measurement of blood glucose concentrations. The inhibitors of PI3K (LY294002 [5 $\mu \mathrm{g}]$ and wortmannin $[0.5 \mu \mathrm{g}])$ or their vehicles ( $1 \%$ methanol and DMSO, respectively) and of MAPK (PD98059 [5 $\mu \mathrm{g}]$ and U0126 [7 $\mu \mathrm{g}]$ ) or their vehicle (DMSO) were administered 10 minutes before insulin, CRF, and their vehicles. Each ICV injection was administered for a duration of 1 minute in a volume of $2 \mu \mathrm{l}$. After ICV administration of experimental agents, mean arterial pressure, HR, and SNA measurements were made every 15 minutes for 6 hours. Blood glucose was measured every 2 hours. At the end of the experiments, CSF and arterial blood were collected. For examination of the effect of ICV insulin on CSF glucose at earlier time points, separate studies were performed in different animals. For collection of CSF, an incision was made to expose the membrane located in the area between the occipital notch and first cervical vertebrae. Once the membrane was exposed, a 23-gauge needle on a 1-ml syringe was then used to puncture the membrane and the CSF was carefully suctioned out immediately after. Rats were then sacrificed by methohexital overdose.

Biochemical studies. Rats were made to fast overnight before ICV insulin $(500 \mathrm{mU})$ and were killed at the indicated time points (see Figure 2) by $\mathrm{CO}_{2}$ asphyxiation. The mediobasal hypothalamus was removed from each rat and was immediately placed in lysis solution $(50 \mathrm{mmol} / \mathrm{l} \mathrm{HEPES}, \mathrm{pH} 7.8$, $137 \mathrm{mmol} / \mathrm{l} \mathrm{NaCl}, 1 \mathrm{mmol} / 1 \mathrm{Na}_{3} V_{4}, 1 \mathrm{mmol} / 1 \mathrm{MgCl}_{2}, 2 \mathrm{mmol} / \mathrm{l}$ EDTA, and $1 \%$ NP-40). After homogenization and centrifugation, the supernatant was removed and stored at $-80^{\circ} \mathrm{C}$. The concentration of total protein was measured with the Bradford method.

For assessment of the effect of insulin on PI3K, $100 \mu \mathrm{g}$ of protein samples were incubated overnight at $4{ }^{\circ} \mathrm{C}$ with anti-IRS- 1 in presence of protein A-sepharose. The immunoprecipitates were washed three times, and collected pellets were resolved by $10 \%$ SDS-PAGE and were electrotransferred overnight to PVDF membranes, which were incubated for 1 hour with specific antibody against PI3K (1:1,000 dilution), and then for 1 hour at room temperature with horseradish peroxidase-linked anti-rabbit immunoglobulin (1:2,000 dilution). For examination of the effect of insulin on MAPK, protein samples $(2 \mu \mathrm{g})$ of homogenized tissues were separated by $10 \%$ SDS-PAGE and were electrotransferred overnight to PVDF membranes. Membranes were incubated for 1 hour with specific antibodies against both p42 and p44 MAPK (1:1,000 dilution) or tyrosine-phosphorylated p42 and p44 MAPK (1:250 dilution), and then for 1 hour at room temperature with horseradish peroxidase-linked anti-rabbit immunoglobulin (1:2000 dilution) or anti-mouse immunoglobulin (1:1000 dilution), respectively. PI3K, p42 and p44 MAPK, and tyrosinephosphorylated $\mathrm{p} 42$ and $\mathrm{p} 44$ MAPK were detected with ECL and film exposure. Image J software was used to quantify band intensity.

Blood glucose and insulin assay. Plasma and CSF glucose concentrations were determined with a glucose analyzer (Yellow Springs Instruments). Plasma and CSF insulin concentrations were measured by radioimmunoassay with commercially available kits (from Linco Inc.).

Data analysis. Because there is significant interindividual variation in baseline SNA, the data for SNA are expressed as percentage change from baseline, with $0 \%$ as baseline. All results are expressed as mean \pm SEM and were analyzed with Student's $t$ test, one- or two-way analysis of variance (ANOVA). When ANOVA reached significance, a post-hoc comparison was made with the Bonferroni test. A $P$ value of less than 0.05 was considered to be statistically significant.

\section{Acknowledgments}

The authors are indebted to Judy Herlein and William Sivitz for assistance in the insulin assay. This work was supported by grants HL44546, HL14388, HL48058, HL61446, and HL55006 from the National Heart, Lung, and Blood Institute. Support by the Heartland Affiliate of American Heart Association to K. Rahmouni (postdoctoral fellowship award $0325567 Z$ ) is gratefully acknowledged.

Received for publication March 29, 2004, and accepted in revised form July 6, 2004.

Address correspondence to: William G. Haynes, Department of Internal Medicine, University of Iowa College of Medicine, Iowa City, Iowa 52242, USA. Phone: (319) 356-8469; Fax: (319) 3537340; E-mail: william-g-haynes@uiowa.edu.
1. Rowe, J.W., et al. 1981. Effect of insulin and glucose infusions on sympathetic nervous system activity in normal man. Diabetes. 30:219-225.

2. Liang, C., et al. 1982. Insulin infusion in conscious dogs. Effects on systemic and coronary hemodynamics, regional blood flows, and plasma catecholamines. J. Clin. Invest. 69:1321-1336.

3. Lembo, G., et al. 1992. Abnormal sympathetic overactivity evoked by insulin in the skeletal muscle of patients with essential hypertension. J. Clin. Invest. 90:24-29.

4. Tomiyama, H., et al. 1992. Blood pressure response to hyperinsulinemia in salt-sensitive and salt-resistant rats. Hypertension. 20:596-600.

5. Anderson, E.A., Hoffman, R.P., Balon, T.W., Sinkey, C.A., and Mark, A.L. 1991. Hyperinsulinemia produces both sympathetic neural activation and vasodilation in normal humans. J. Clin. Invest. 87:2246-2252.

6. Vollenweider, L., et al. 1995. Insulin-induced sympathetic activation and vasodilation in skeletal muscle. Effects of insulin resistance in lean subjects. Diabetes. 44:641-645.

7. Morgan, D.A., Balon, T.W., Ginsberg, B.H., and Mark, A.L. 1993. Nonuniform regional sympathetic nerve responses to hyperinsulinemia in rats. $A m$. J. Physiol. 264:R423-R427.

8. Muntzel, M.S., Morgan, D.A., Mark, A.L., and Johnson, A.K. 1994. Intracerebroventricular insulin produces nonuniform regional increases in sympathetic nerve activity. Am. J. Physiol. 267:R1350-R1355.

9. Muntzel, M., Beltz, T., Mark, A.L., and Johnson, A.K. 1994. Anteroventral third ventricle lesions abolish lumbar sympathetic responses to insulin. Hypertension. 23:1059-1062.

10. Havrankova, J., Roth, J., and Brownstein, M. 1978. Insulin receptors are widely distributed in the central nervous system of the rat. Nature. 272:827-829.

11. Werther, G.A., et al. 1987. Localization and characterization of insulin receptors in rat brain and pituitary gland using in vitro autoradiography and computerized densitometry. Endocrinology.
121:1562-1570.

12. Zhao, W.Q., and Alkon, D.L. 2001. Role of insulin and insulin receptor in learning and memory. Mol. Cell. Endocrinol. 177:125-134.

13. Saltiel, A.R., and Kahn, C.R. 2001. Insulin signalling and the regulation of glucose and lipid metabolism. Nature. 414:799-806.

14. Baumann, C.A., and Saltiel, A.R. 2001. Spatial compartmentalization of signal transduction in insulin action. Bioessays. 23:215-222.

15. Shepherd, P.R., Withers, D.J., and Siddle, K. 1998. Phosphoinositide 3-kinase: the key switch mechanism in insulin signalling. Biochem. J. 333:471-490.

16. Correia, M.L., et al. 2001. Role of corticotrophinreleasing factor in effects of leptin on sympathetic nerve activity and arterial pressure. Hypertension. 38:384-388.

17. Berne, C., Fagius, J., Pollare, T., and Hjemdahl, P. 1992. The sympathetic response to euglycaemic hyperinsulinaemia. Evidence from microelectrode nerve recordings in healthy subjects. Diabetologia. 
35:873-879

18. Scherrer, U., and Sartori, C. 1997. Insulin as a vascular and sympathoexcitatory hormone: implications for blood pressure regulation, insulin sensitivity, and cardiovascular morbidity. Circulation. 96:4104-4113.

19. Benoit, S.C., Clegg, D.J., Seeley, R.J., and Woods, S.C. 2004. Insulin and leptin as adiposity signals. Recent Prog. Horm. Res. 59:267-285.

20. Obici, S., Feng, Z., Karkanias, G., Baskin, D.G., and Rossetti, L. 2002. Decreasing hypothalamic insulin receptors causes hyperphagia and insulin resistance in rats. Nat. Neurosci. 5:566-572.

21. Bruning, J.C., et al. 2000. Role of brain insulin receptor in control of body weight and reproduction. Science. 289:2122-2125.

22. Menendez, J.A., and Atrens, D.M. 1991. Insulin and the paraventricular hypothalamus: modulation of energy balance. Brain Res. 555:193-201.

23. Geloen, A., and Trayhurn, P. 1990. Regulation of the level of uncoupling protein in brown adipose tissue by insulin requires the mediation of the sympathetic nervous system. FEBS Lett. 267:265-267.

24. Obici, S., Zhang, B.B., Karkanias, G., and Rossetti, L. 2002. Hypothalamic insulin signaling is required for inhibition of glucose production. Nat. Med. 8:1376-1382.

25. Szabo, A.J., and Szabo, O. 1983. Insulin injected into CNS structures or into the carotid artery: effect on carbohydrate homeostasis of the intact animal. Adv. Metab. Disord. 10:385-400.

26. Szabo, O., and Szabo, A.J. 1975. Studies on the nature and mode of action of the insulin-sensitive glucoregulator receptor in the central nervous system. Diabetes. 24:328-336.

27. Edwards, J.G., and Tipton, C.M. 1989. Influences of exogenous insulin on arterial blood pressure measurements of the rat. J. Appl. Physiol. 67:2335-2342.

28. Meehan, W.P., Buchanan, T.A., and Hsueh, W. 1994. Chronic insulin administration elevates blood pressure in rats. Hypertension. 23:1012-1017.

29. Rocchini, A.P., et al. 1989. Insulin and renal sodium retention in obese adolescents. Hypertension. 14:367-374.

30. Porter, J.P. 1994. Effect of intrahypothalamic insulin on sympathetic nervous function in rats drinking a high-sucrose solution. Am. J. Physiol. 266:R1463-R1469.

31. Landsberg, L. 2001. Insulin-mediated sympathetic stimulation: role in the pathogenesis of obesityrelated hypertension (or, how insulin affects blood pressure, and why). J. Hypertens. 19:523-528.

32. Spanswick, D., Smith, M.A., Mirshamsi, S., Routh, V.H., and Ashford, M.L. 2000. Insulin activates ATPsensitive $\mathrm{K}^{+}$channels in hypothalamic neurons of lean, but not obese rats. Nat. Neurosci. 3:757-758.

33. Niswender, K.D., et al. 2003. Insulin activation of phosphatidylinositol 3-kinase in the hypothalamic arcuate nucleus: a key mediator of insulin-induced anorexia. Diabetes. 52:227-231.
34. Carvalheira, J.B., et al. 2003. Selective impairment of insulin signalling in the hypothalamus of obese Zucker rats. Diabetologia. 46:1629-1640.

35. Benoit, S.C., et al. 2002. The catabolic action of insulin in the brain is mediated by melanocortins. J. Neurosci. 22:9048-9052.

36. Schwartz, M.W., et al. 1992. Inhibition of hypothalamic neuropeptide $Y$ gene expression by insulin. Endocrinology. 130:3608-3616.

37. Rahmouni, K., Haynes, W.G., Morgan, D.A., and Mark, A.L. 2003. Role of melanocortin-4 receptors in mediating renal sympathoactivation to leptin and insulin. J. Neurosci. 23:5998-6004.

38. Davies, S.P., Reddy, H., Caivano, M., and Cohen, P. 2000. Specificity and mechanism of action of some commonly used protein kinase inhibitors. Biochem. J. 351:95-105.

39. Alessi, D.R., Cuenda, A., Cohen, P., Dudley, D.T. and Saltiel, A.R. 1995. PD 098059 is a specific inhibitor of the activation of mitogen-activated protein kinase kinase in vitro and in vivo. J. Biol. Chem. 270:27489-27494.

40. Favata, M.F., et al. 1998. Identification of a novel inhibitor of mitogen-activated protein kinase kinase. J. Biol. Chem. 273:18623-18632.

41. Morgan, D.A., Thoren, P., Staudt, S.M., and Mark, A.L. 1988. Differential effects of anesthesia on heart rate (HR), renal (RSNA), and lumbar sympathetic nerve activity (LSNA) in the rat [abstract]. FASEB J. 2:A1690. 